\title{
HETEROGENEOUS CONDUCTING POLYPYRROLE BASED SENSING COMPOSITES
}

\author{
Vineet V. Karambelkar", S.N. Paul* and J.D. Ekhe ${ }^{\dagger}$ \\ "Department of Metallurgical and Materials Engineering, Visvesvaraya National Institute of \\ Technology, Nagpur-10, India. \\ ${ }^{\dagger}$ Department of Chemistry, Visvesvaraya National Institute of Technology, Nagpur-10, India. \\ *E-mail: vineet.karambelkar@gmail.com
}

\begin{abstract}
Polypyrrole heterogeneous composites were formulated which exhibited electrical conductivity. Lead Zirconate Titanate piezoceramic powder was used as a doping agent and the resulting polymer was obtained through emulsion polymerization. Electrical conductivity up to the order of $4.7 \mathrm{~S} / \mathrm{cm}$ was obtained in these composites. It was observed that even though the percentage of Tin metal was increased in these composites, a decrease in the concentration of piezoceramic powder decreased their electrical conductivity. Greater concentrations of piezoceramic powder yielded greater conductivities. This observation confirmed the heterogeneous nature of Polypyrrole based composites.
\end{abstract}

Keywords: Polypyrrole; Heterogeneous Composite; Sensor.

@ RASĀYAN. All rights reserved

\section{INTRODUCTION}

Heterogeneity in conducting polymers or fully organic heterogeneous conducting systems is an emerging thrust area of polymer technology wherein characteristic temperature dependence on the conductivity of the conducting polymers with an increased doping level is proficiently demonstrated by Gui et al. ${ }^{1}$ Most important characteristic of the conductivity of the organic conducting polymers is its remarkably large magnitude for materials with low carrier density and significant disorder, Asadi et al. ${ }^{2} \mathrm{Myriad}$ applications of these polymers include Nanoelectromechanical devices (NEMS), Microelectromechanical devices (MEMS) and nowadays these polymers have become the backbone of optoelectronics, Ince et. $a l .{ }^{3}$ Polypyrrole, itself a conducting polymer par excellence, has gained worldwide attention in recent times, owing to very high electrical conductivity displayed by the pure polymer, which can go as high as 2000 S/cm, Qi et. al. ${ }^{4}$ Dealing with such a highly conductive polymer offers a great platform for advanced research and the current research work attempts to tap the property of heterogeneity in this magnificent polymer. The main advantage with Polypyrrole is its chemical stability which remains unaltered over a period of time and this property helps to develop novel heterogeneous composites having wide industrial applications. High-performance supercapacitors based on Polypyrrole/ $\mathrm{NiCO}_{2} \mathrm{O}_{4}$ nano wire arrays on $\mathrm{Ni}$ foam is an excellent example of the application of Polypyrrole in battery technology, $\mathrm{Hu}$ et al..$^{5}$ The comparatively low electrical conductivity of nano-sized $\mathrm{Al}_{2} \mathrm{O}_{3}$ doped with PVP (polyvinyl pyrrolidine) based solid polymer electrolyte was found to be $1.22 \times 10^{-6} \mathrm{~S} / \mathrm{cm}$ at room temperature for $85: 15$ compositions having low activation energy. ${ }^{6}$ Polyaniline (PAni), doped with Sulphuric acid was synthesized by the electrochemical method at $1.7 \mathrm{~V}$ yielded electrical conductivity of $0.83 \mathrm{~S} / \mathrm{cm}^{7}$ Neodymium oxide $\left(\mathrm{Nd}_{2} \mathrm{O}_{3}\right)$ /polyvinyl alcohol composites were prepared by sol gel method, and an increase in impedance values was observed with increasing concentration of polyvinyl alcohol, indicating corrosion protection through this composites. ${ }^{8}$

In this paper, Polypyrrole/Piezoceramic composites have been shown to have sensing property towards a low-temperature change in the range of $303 \mathrm{~K}-373 \mathrm{~K}$ with possible application as a piezoelectric actuator. 


\section{EXPERIMENTAL}

Pyrrole monomer (Acros Organics) was distilled prior the polymerization process and the piezoceramic powder, Lead Zirconate Titanate with a particle size of $950 \mathrm{~nm}$ was used as is. Tin metal powder (Loba Chemie) with a mesh size of 325 meshes was also used as is. Sodium Lauryl Sulfate (Sigma Aldrich) and Potassium Persulfate (Sigma Aldrich) were also used as received. Resistance measurements were taken on a two point probe digital multimeter.

An emulsion of sodium lauryl sulfate surfactant $(3.7 \mathrm{~g})$ and potassium persulfate oxygen ion donating initiator $(6.0 \mathrm{~g})$ was prepared in a $250 \mathrm{ml}$ beaker and pyrrole monomer $(5.5 \mathrm{ml})$ along with piezoceramic powder and the Tin metal powder was added in various percentages as given in Table-1. The resulting grayish black polymer was filtered using Whatman Filter Paper Number 40 and dried in a hot air oven at $150^{\circ} \mathrm{C}$. The polymer was subsequently weighed till three constant weights were obtained.

Three piezoceramic composites with varying percentages of Lead Zirconate Titanate and Tin metal powder were formulated as given in Table-1.

Table-1: Piezoceramic composite mixtures

\begin{tabular}{c|c|c}
\hline Composite Number & Tin Powder (\%) & Piezoceramic Powder (\%) \\
\hline 1 & 0.5 & 7.2 \\
\hline 2 & 1.0 & 9.8 \\
\hline 3 & 2.0 & 5.5 \\
\hline
\end{tabular}

The synthesized polymer samples were then pressed in a hydraulic pellet press at 7.2 tons of pressure to yield pellets of uniform thickness of $1 \mathrm{~mm}$. Resistance in Ohms $(\Omega)$ of these pressed pellets was measured and conductivity was calculated using the relation $\mathrm{C}=\Omega^{-1}$. Table 2 gives the conductivities obtained for these pressed pellets.

Table-2: Conductivities of Polypyrrole/Piezoceramic powder composites

\begin{tabular}{c|c}
\hline Composite Number & Conductivity $(\mathrm{S} / \mathrm{cm})$ \\
\hline 1 & 3.37 \\
\hline 2 & 4.77 \\
\hline 3 & $10^{-4}$ \\
\hline
\end{tabular}

\section{RESULTS AND DISCUSSION}

It was observed that conductivity decreased from $4.77 \mathrm{~S} / \mathrm{cm}$ to $10^{-4} \mathrm{~S} / \mathrm{cm}$ irrespective of the increase in the percentage of Tin metal powder. The decrease in the percentage of piezoceramic powder lowered the conductivity, whereas an increase in the piezoceramic powder caused an increase in the conductivity values. This observation explains the true heterogeneous nature of these composites.

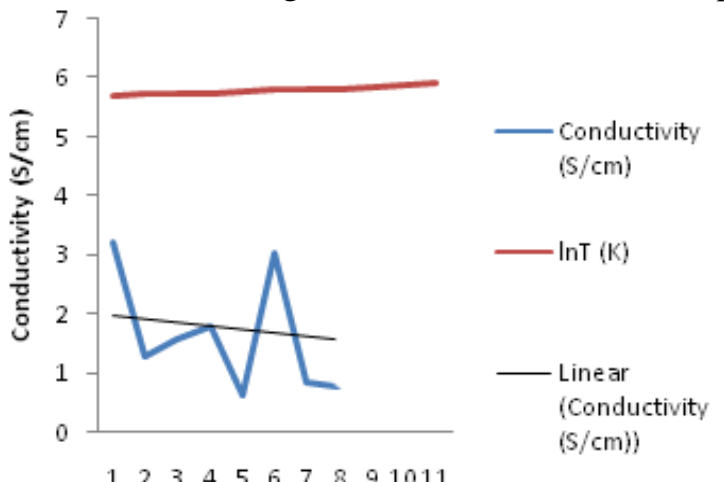

$\ln T(K)$

(a) 
RASĀYAN J. Chem.

Vol. 10 | No. 3 |832 - 837 | July - September | 2017

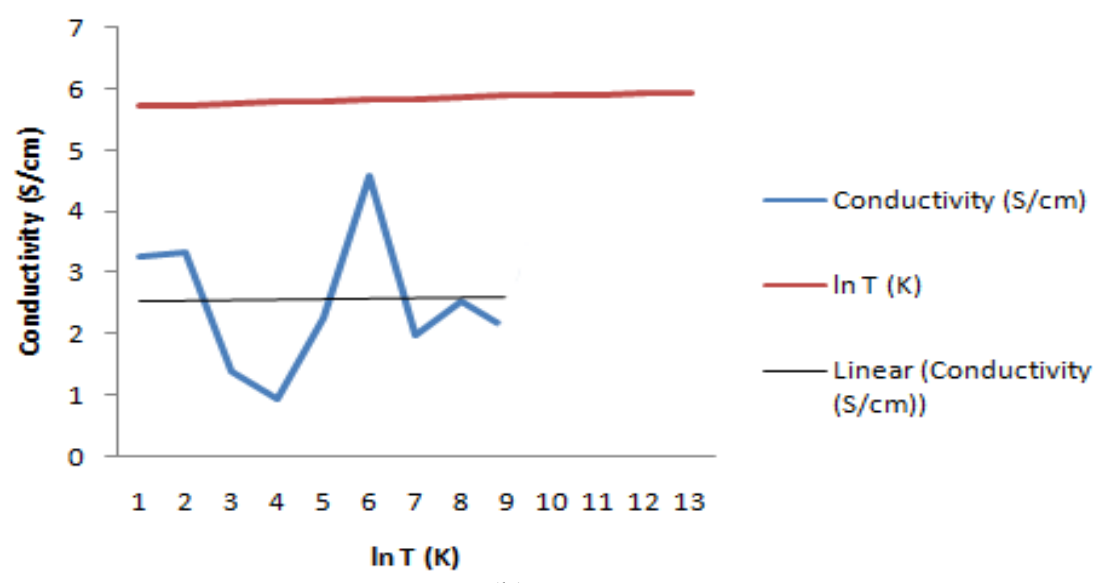

(b)

Fig.-1: (a) Effect of temperature on the conductivity of composite 1 and (b) Effect of temperature on the conductivity of composite 2

The resulting composites were found to have excellent chemical stability with very low level of contamination and their conductivity remained unaltered even after long storage times ( three months). Figure-1 illustrates the effect of temperature on the conductivity of Polypyrrole/Piezoceramic composite 1 and 2 respectively. Figure- 2 shows the effect of temperature on composite 3.

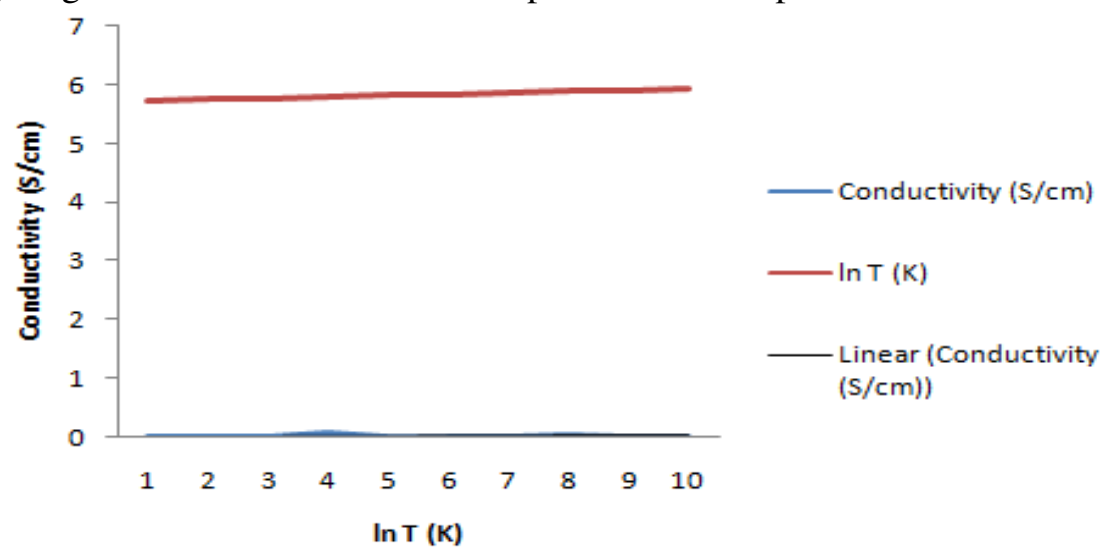

Fig.-2: Effect of temperature on the conductivity of composite 3

It was observed that the composite number 2 with $9.8 \%$ of piezoceramic powder showed maximum conductivity of $4.77 \mathrm{~S} / \mathrm{cm}$ and the conductivity decreased when the concentration of piezoceramic powder was dropped even though Tin metal powder concentration was increased. The actual Polypyrrole/Piezoceramic composite is shown in Fig.-3.

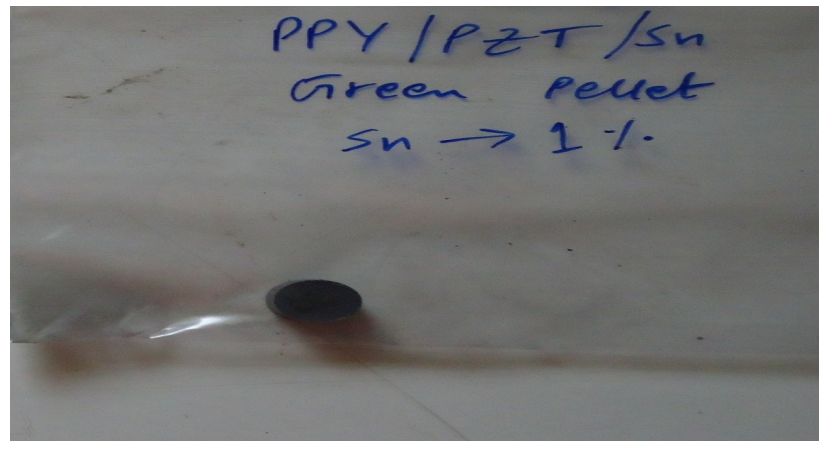

Fig.-3: Actual Polypyrrole/Piezoceramic composite 
RASĀYAN J. Chem.

Vol. 10 | No. 3 |832 - 837 | July - September | 2017

A comparison between the applied voltage and the corresponding temperatures is depicted in Fig.- 4 of the composites 1, 2 and 3 respectively.

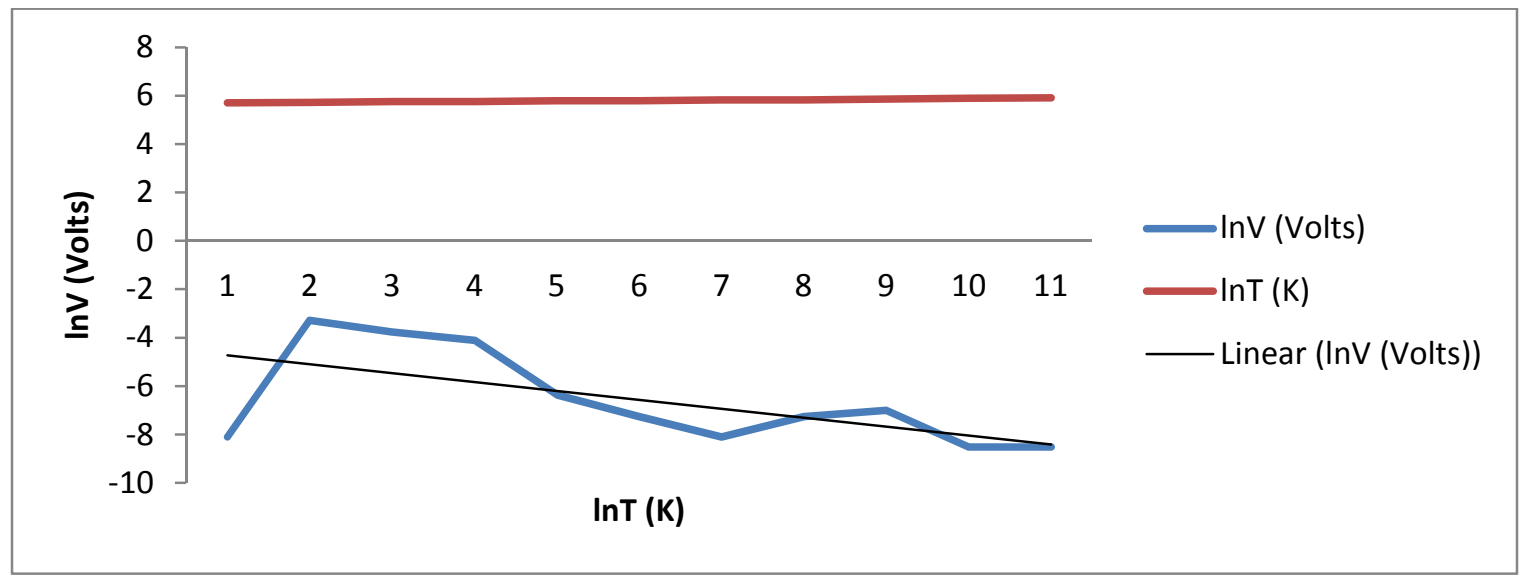

(a)

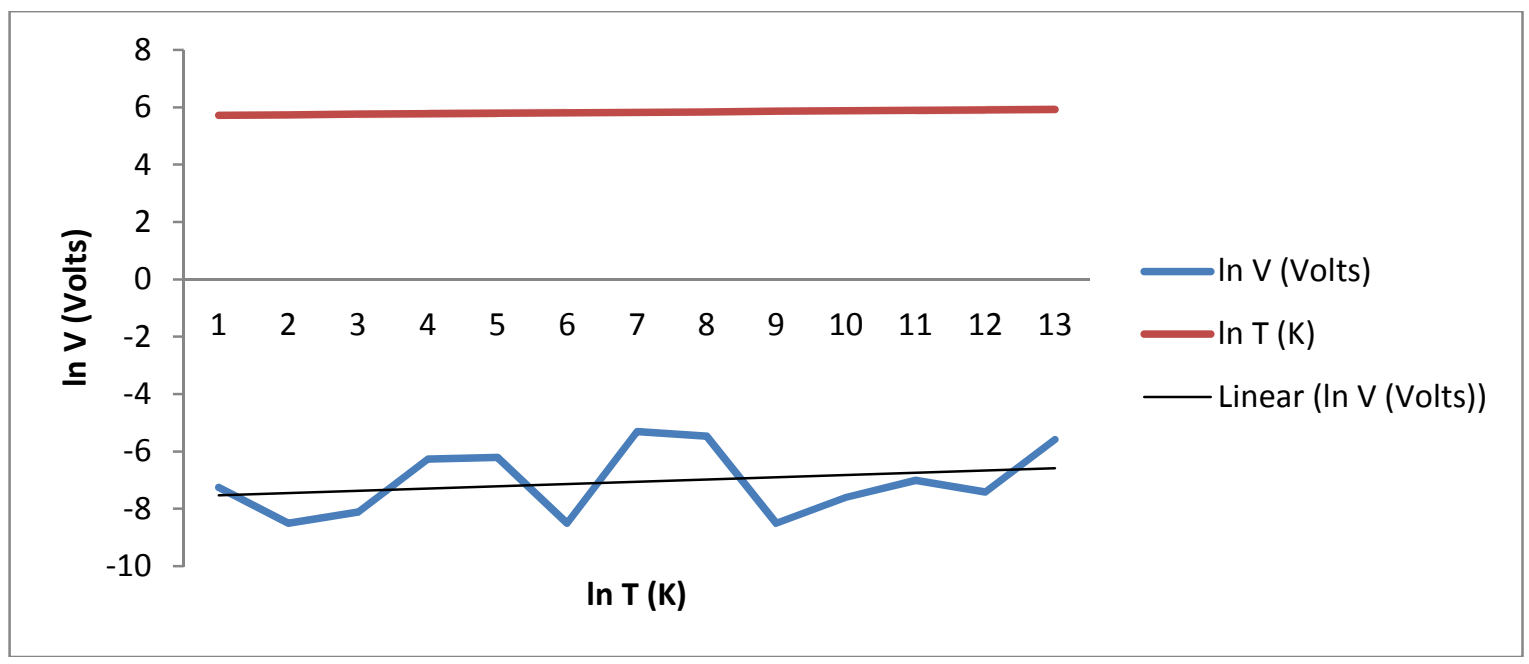

(b)

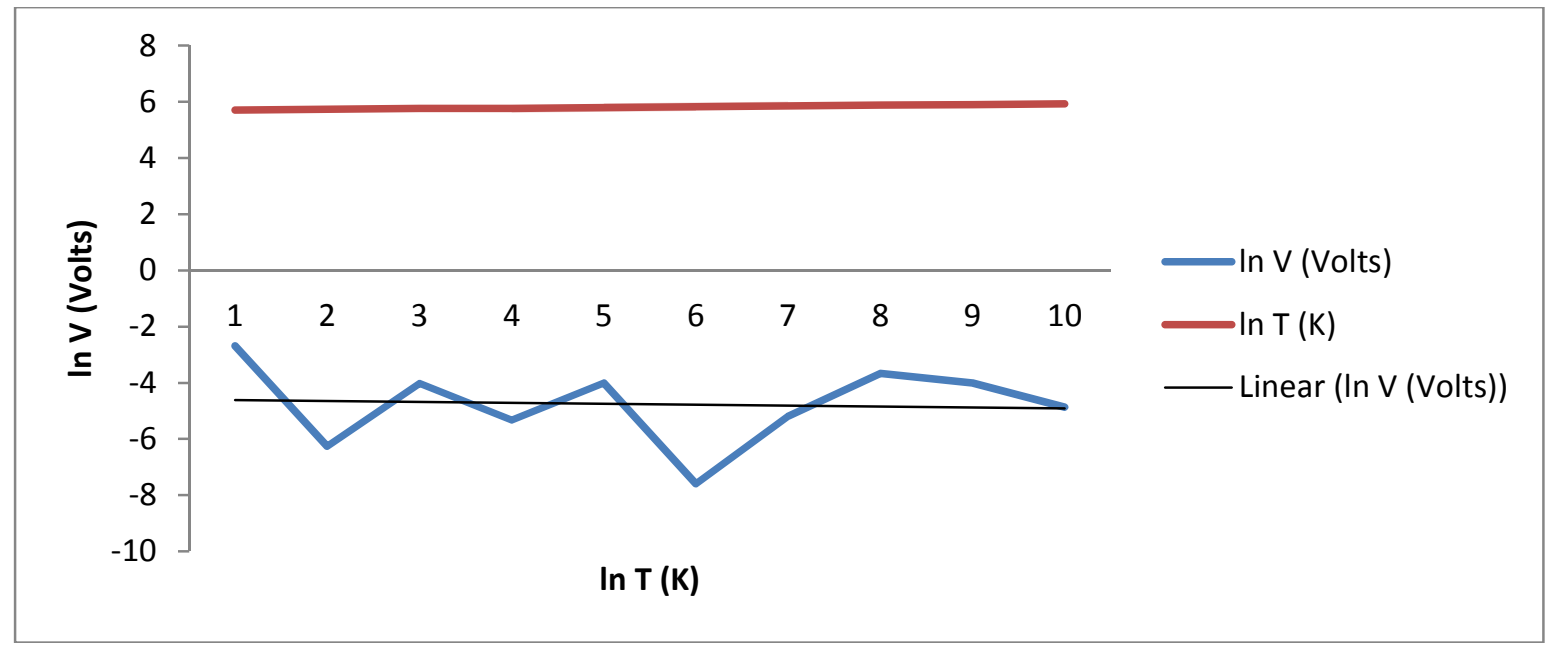

(c)

Fig.-4: Applied voltage against temperature of composites: (a) 1 (b) 2 and (c) 3 
RASĀYAN J. Chem.

Vol. 10 | No. 3 |832 - 837 | July - September | 2017

It was evident from Fig.-4(b) that even a slight temperature change produced a weak voltage signal, and as the temperature range increased till $373 \mathrm{~K}$ the voltage signal became strong, reached a maximum and then decreased. This was the sample in which percentage of piezoceramic powder was more as compared to samples in 4 (a) and 4 (c). However, in Fig.-4 (a) and 4 (c) voltage signal consistently decreased with a rise in temperatures, where the percentage of the piezoceramic powder was lesser, in spite of the increase in the percentage of Tin metal powder. This observation clearly indicated heterogeneous nature of the Polypyrrole/Piezoceramic composites and the success of the experiment. Characterization of these Polypyrrole/Piezoceramic composites by SEM micrograph studies revealed uniform deposition of the piezoceramic powder layer over the polymer along with Tin metal powder. Figure-5 depicts the SEM micrograph of the Polypyrrole/Piezoceramic composite.

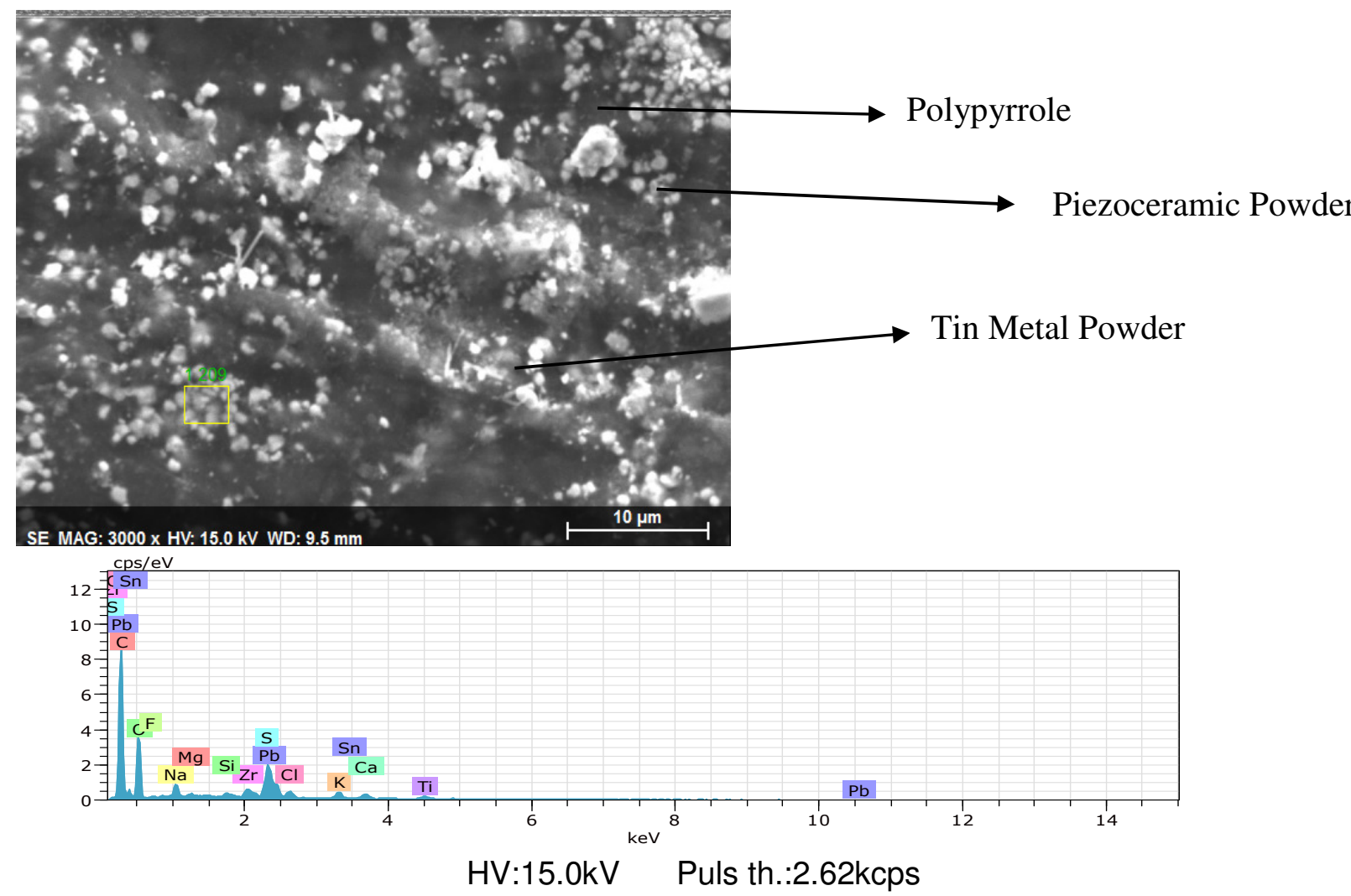

\begin{tabular}{|c|c|c|c|c|c|c|}
\hline El & AN & Series & $\begin{array}{l}\text { unn. C } \\
{\left[w t . \frac{\circ}{0}\right]}\end{array}$ & $\begin{array}{l}\text { norm.c } \\
{\left[w t . \frac{\circ}{\circ}\right]}\end{array}$ & $\begin{array}{l}\text { om. C } \\
{\left[a t . \frac{\circ}{o}\right]}\end{array}$ & $\begin{array}{l}\text { igma) } \\
{\left[w t . \frac{\circ}{\circ}\right]}\end{array}$ \\
\hline C & 6 & K-series & 32.19 & 48.75 & 64.06 & 4.01 \\
\hline O & 8 & K-series & 20.17 & 30.54 & 30.13 & 2.74 \\
\hline $\mathrm{Pb}$ & 82 & M-series & 6.18 & 9.36 & 0.71 & 0.26 \\
\hline & 16 & K-series & 1.57 & 2.37 & 1.17 & 0.09 \\
\hline Zr & 40 & L-series & 1.19 & 1.81 & 0.31 & 0.08 \\
\hline $\mathrm{Na}$ & 11 & K-series & 1.12 & 1.70 & 1.17 & 0.10 \\
\hline K & 19 & K-series & 0.88 & 1.33 & 0.54 & 0.06 \\
\hline $\mathrm{Ca}$ & 20 & K-series & 0.85 & 1.29 & 0.51 & 0.06 \\
\hline $\mathrm{Ti}$ & 22 & K-series & 0.81 & 1.22 & 0.40 & 0.06 \\
\hline $\mathrm{Cl}$ & 17 & K-series & 0.55 & 0.84 & 0.37 & 0.05 \\
\hline $\mathrm{F}$ & 9 & K-series & 0.44 & 0.66 & 0.55 & 0.16 \\
\hline & 12 & K-series & 0.06 & 0.09 & 0.06 & 0.03 \\
\hline
\end{tabular}


RASĀYAN J. Chem.

Vol. 10 | No. 3 |832 - 837 | July - September | 2017

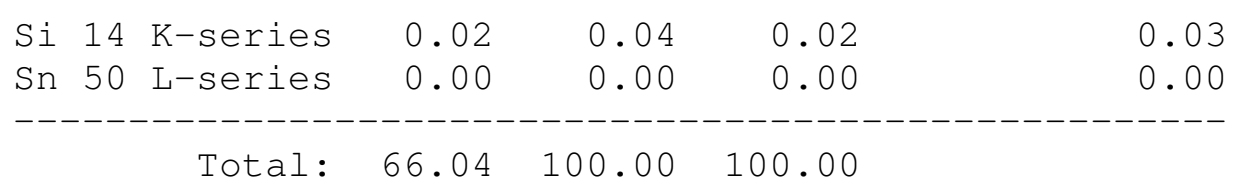

Fig.-5: SEM Micrograph with EDX data of the Polypyrrole/Piezoceramic composite

\section{CONCLUSION}

Truly heterogeneous Polypyrrole/Piezoceramic sensing composites were formulated with the possible application in the area of piezoelectric actuators. The main advantage of the process through which these composites were generated was the facile nature of polymerization and extremely low levels of contamination. It was evident from this work that the heterogeneous nature of these composites depended upon the percentage of the piezoceramic powder used.

\section{REFERENCES}

1. Z. Gui, J. Duay, J. Hu, S. B. Lee, Physical Chemistry Chemical Physics, 16,12332(2014).

2. K. Asadi, A.J. Kronemeijer, T. Cramer, L.J.A. Coster, P.W.M. Blom, D.M. de Leeuw, Nature Communications, 4, 1710(2013).

3. G.O. Ince, A.M. Coclite, K.K. Gleason, Reports on Progress in Physics, 75, 016501(2012).

4. G. Qi, L. Huang, H. Wang, Chemical Communications, 48 ,8246(2012).

5. J. Hu, M. Li, F. Lv, M. Yang, P. Tao, Y, Tang, H. Liu, Z, Lu, Journal of Power Sources, 294,120(2015).

6. S. Basha, G. Sundari, K. Kumar, K. Reddy, M. Rao, RASĀYAN J. Chem., 10(1), 279(2017).

7. G. Umadevi, V. Ponnusamy, M. Paramsivam, S. Palaniswami, RASĀYAN J. Chem., 3(1), 194(2010).

8. N. Venkatesan, P. Kamaraj, S. Devikala, M. Arthanareeswari, , RASĀYAN J. Chem., 8(3), 321(2015).

[RJC-1687/2017] 